\title{
Current Fluctuations for the Totally Asymmetric Simple Exclusion Process
}

\author{
Michael Prähofer and Herbert Spohn \\ Zentrum Mathematik und Physik Department, TU München, \\ D-80290 München, Germany \\ emails: praehofer@ma.tum.de, spohn@ma.tum.de \\ October 28, 2018
}

\begin{abstract}
The time-integrated current of the TASEP has non-Gaussian fluctuations of order $t^{1 / 3}$. The recently discovered connection to random matrices and the Painlevé II RiemannHilbert problem provides a technique through which we obtain the probability distribution of the current fluctuations, in particular their dependence on initial conditions, and the stationary two-point function. Some open problems are explained.
\end{abstract}




\section{Introduction}

The asymmetric simple exclusion process (ASEP) has become a paradigm for nonreversible stochastic particle systems. In this note we will consider the particular case of one dimension and right jumps only (TASEP). The TASEP has state space $\{0,1\}^{\mathbb{Z}}$ whose elements, $\eta$, are particle configurations with $\eta_{j}=0,1$ for $j \in \mathbb{Z}$. $\eta_{j}=1$ means a particle at site $j$ and $\eta_{j}=0$ means no particle, resp. a hole, at site $j$. The stochastic dynamics is governed by the following jump rule: independently each particle jumps with rate 1, i.e. after an exponential waiting time with mean 1, to the right neighboring site, provided it is empty. Our rule defines the Markov jump process $\eta_{t}, t \geq 0$. $\eta_{0}$ is the starting configuration which may be random.

For the purpose of the introduction let us assume that $\eta_{0}$ is distributed according to the Bernoulli measure $\mu_{1 / 2}$, i.e. $\eta_{0, j}, j \in \mathbb{Z}$, are independent with $\mathbb{P}\left(\eta_{0, j}=1\right)=\frac{1}{2}$. Since $\mu_{1 / 2}$ is a stationary measure for the TASEP, the process $\eta_{t, j}, t \in \mathbb{R}, j \in \mathbb{Z}$, is stationary in space and time. We are interested in

$N_{t}=$ number of particles which have crossed the bond $(0,1)$ up to time $t$.

Clearly, $\frac{d}{d t} N_{t}$ is the current across the origin, hence our title. By stationarity,

$$
\mathbb{E}\left(N_{t}\right)=\frac{1}{4} t
$$

and the real issue are the fluctuations of

$$
N_{t}-\frac{1}{4} t
$$

To convince the reader that the current fluctuations have something interesting to offer, we first study the large deviations of $N_{t}$. For the lower tail we consider $\mathbb{P}\left(N_{t} \leq a t\right)$ with $a<\frac{1}{4}$. To reduce the current it suffices to let a single particle move more slowly. The other particles will then pile up behind. Therefore we expect

$$
\mathbb{P}\left(N_{t} \leq a t\right) \simeq e^{-g_{-}(a) t}, \quad a \leq \frac{1}{4},
$$

for large $t$ with $g_{-} \geq 0$ and $g_{-}\left(\frac{1}{4}\right)=0$. On the other hand for the upper tail, $\mathbb{P}\left(N_{t} \geq a t\right), a>\frac{1}{4}$, the current must be increased, which requires order $t$ particles to jump faster. Therefore

$$
\mathbb{P}\left(N_{t} \geq a t\right) \simeq e^{-g_{+}(a) t^{2}}, \quad a \geq \frac{1}{4},
$$

for large $t$ with $g_{+} \geq 0$ and $g_{+}\left(\frac{1}{4}\right)=0$. Since the large deviations have different order of magnitude above and below the mean, we must be outside the domain of the central limit theorem.

A more detailed analysis shows that $g_{-}(a) \simeq c_{-}\left|a-\frac{1}{4}\right|^{3 / 2}$ and $g_{+}(a) \simeq c_{+}\left|a-\frac{1}{4}\right|^{3}$ for $a$ close to $\frac{1}{4}$ 11, 2], also [3, 4] for the PNG model. Extrapolating beyond the validity of the large deviation result, we conclude that for the lower tail

$$
\mathbb{P}\left(N_{t}-\frac{1}{4} t \simeq x\right) \simeq e^{-c_{-}\left|x / t^{1 / 3}\right|^{3 / 2}} \quad \text { for } x \ll-1
$$


and for the upper tail

$$
\mathbb{P}\left(N_{t}-\frac{1}{4} t \simeq x\right) \simeq e^{-c_{+}\left|x / t^{1 / 3}\right|^{3}} \quad \text { for } x \gg 1
$$

On this basis the fluctuations should be on the scale $t^{1 / 3}$ and

$$
N_{t}-\frac{1}{4} t \cong t^{1 / 3} \xi
$$

for large $t . \quad \xi$ is a nondegenerate random variable with a distribution whose lower tail is $\exp \left(-c_{-}|x|^{3 / 2}\right)$ and whose upper tail is $\exp \left(-c_{+}|x|^{3}\right)$.

The goal of our note is to explain how (1.7) and related quantities like the stationary two-point function, $\mathbb{E}\left(\eta_{0,0} \eta_{t, j}\right)-\frac{1}{4}$, can be mapped to a last passage percolation problem with boundary conditions and possibly defect lines. The asymptotic analysis of such last passage percolations has been carried out by Baik and Rains [5] and we will make contact with their work towards the end.

\section{Last passage percolation with boundary conditions}

We generalize the set up of the introduction by taking instead of $\mu_{1 / 2}$ the Bernoulli $\mu_{\rho_{-}, \rho_{+}}$ as starting measure, according to which the $\eta_{0, j}$ 's are independent with $\mathbb{P}\left(\eta_{0, j}=1\right)=\rho_{-}$for $j \leq 0$ and $\mathbb{P}\left(\eta_{0, j}=1\right)=\rho_{+}$for $j \geq 1$. $\mu_{1 / 2}$ is the special case with $\rho_{-}=\frac{1}{2}=\rho_{+}$. Liggett [6] determines the law of $\eta_{t}$ as $t \rightarrow \infty$. We will come back to his result. Johansson [2] maps the special case $\rho_{-}=1, \rho_{+}=0$ to a last passage percolation. We extend his result to arbitrary $0 \leq \rho_{-}, \rho_{+} \leq 1$.

Let us first assign to an ASEP configuration $\eta_{t, j}$ the height function

$$
h_{t}(j)= \begin{cases}2 N_{t}+\sum_{i=1}^{j}\left(1-2 \eta_{t, i}\right), & j \geq 1, \\ 2 N_{t}, & j=0, \\ 2 N_{t}-\sum_{i=j+1}^{0}\left(1-2 \eta_{t, i}\right), & j \leq-1 .\end{cases}
$$

By fiat $h_{0}(0)=0 . h_{t}(j)$ is even at even and odd at odd sites. We will establish that the distribution of the heights can be obtained from a last passage percolation problem on the positive quadrant $\mathbb{N} \times \mathbb{N}$ with suitable boundary conditions.

To each site $(i, j) \in \mathbb{N}_{0}^{2}$ we associate a random variable $w(i, j)$. Let $\zeta_{+}$be geometric with parameter $1-\rho_{+}, \mathbb{P}\left(\zeta_{+}=n\right)=\rho_{+}\left(1-\rho_{+}\right)^{n}$, and independently let $\zeta_{-}$be geometric with parameter $\rho_{-}, \mathbb{P}\left(\zeta_{-}=n\right)=\left(1-\rho_{-}\right) \rho_{-}^{n}, n=0,1, \ldots$ The $\left\{w(i, j) \mid(i, j) \in \mathbb{N}_{0}^{2}\right\}$ are independent 
with distributions:

$$
\begin{aligned}
& w(i, j) \quad \text { is exponential with mean } 1, i, j \geq 1, \\
& w(0,0)=0 \\
& w(j, 0)=0, \quad 0 \leq j \leq \zeta_{+} \\
& w(j, 0) \quad \text { is exponential with mean }\left(1-\rho_{+}\right)^{-1}, j>\zeta_{+} \\
& w(0, j)=0, \quad 0 \leq j \leq \zeta_{-}, \\
& w(0, j) \quad \text { is exponential with mean } \rho_{-}^{-1}, j>\zeta_{-}
\end{aligned}
$$

Let $\Omega_{m, n}$ be the set of all up/right paths on $\mathbb{N}_{0}^{2}$ starting at $(0,0)$ with end point $(m, n)$. To each path $\omega \in \Omega_{m, n}$ we associate the passage time

$$
T(\omega)=\sum_{(i, j) \in \omega} w(i, j)
$$

Then the last passage time, from $(0,0)$ to $(m, n)$, is given by

$$
G(m, n)=\max _{\omega \in \Omega_{m, n}} T(\omega)
$$

In the more physical parlance, $\omega$ is a directed polymer with end points $(0,0)$ and $(m, n)$. Each site carries the energy $-w(i, j)$. The energy of the polymer $\omega,-T(\omega)$, is the sum of the site energies along the polymer. Thus $-G(m, n)$ is the minimal energy, or ground state energy, of the directed polymer.

By construction $G(m, n)$ is nondecreasing in both arguments. Therefore the level sets of $G$ define a height function which we denote by $\tilde{h}_{t}(j)$. More precisely let $A_{t}=\{(m, n) \mid m, n \geq 1$, $G(m, n) \leq t\}$ as a random set. $A_{t}$ is bordered by $\tilde{h}_{t}$ according to $A_{t}=\{(m, n) \mid 2 \leq m+n \leq$ $\left.\tilde{h}_{t}(m-n)\right\}$.

Theorem 2.1 In the sense of joint distributions we have

$$
h_{t}(j)=\tilde{h}_{t}(j) \quad \text { for } \quad|j| \leq h_{t}(j) .
$$

Proof: The idea is to rotate the TASEP height configuration by $-\pi / 4$ relative to the origin and to identify the resulting height differences to the right as a zero range process. Similarly we rotate the TASEP height configuration by $\pi / 4$ relative to the origin and identify the resulting height differences to the left as a zero range process. We start with the former.

We define the right dynamics by setting $\eta_{t, 0}=1$ for all $t \geq 0$. The $\eta_{t, j}, j \geq 1$, follow the TASEP rule. For given $\eta_{t, j}$ let $\zeta_{t, j}, j \geq 1$, be the interparticle distances to the right. Thus if $\eta_{t, 1}=1$, then $\zeta_{t, 1}=0$, since the origin is always occupied. Since the starting measure is Bernoulli $\rho_{+}$for $j \geq 1$, at time $t=0$, the $\left\{\zeta_{0, j}\right\}_{j \geq 1}$ are independently geometrically distributed with parameter $1-\rho_{+} \cdot \zeta_{t, j}, j \geq 1$, is a zero range process where the only allowed transitions are $\left(\zeta_{t, j}, \zeta_{t, j+1}\right) \rightarrow\left(\zeta_{t, j}+1, \zeta_{t, j+1}-1\right)$ for $j=1,2, \ldots$ and they occur with rate 1 provided 
$\zeta_{t, j+1}>0 . \zeta_{t, 1}$ increases in units of 1 and $w(j, 0), j=1,2, \ldots$, are the successive waiting times of $\zeta_{t, 1}$. At $t=0, \zeta_{0,1}$ is geometrically distributed as $\zeta_{+}$. Thus $w(j, 0)=0$ for $1 \leq j \leq \zeta_{+}$. The zero range process $\zeta_{t, j}, j \geq 2$, is stationary. By Burke's theorem [7] the current from 2 to 1 is Poisson with intensity $1-\rho_{+}$. Thus $w(j, 0)$ are independent exponentials for $j>\zeta_{+}$.

For the left boundary we only have to interchange particles and holes. Holes jump to the left only. The left hole dynamics is defined by setting $\eta_{t, 1}=0$ for all $t \geq 0$. The holes $1-\eta_{t, j}$,

$j \leq 0$, follow the TASEP rule. $\zeta_{t, j}, j \leq 0$, are the interhole distances. $\zeta_{t, 0}$ increases in units of 1 and $w(0, j), j=1,2, \ldots$, are the successive waiting times of $\zeta_{t, 0}$. At $t=0, \zeta_{0,0}$ is geometrically distributed as $\zeta$ and $t \mapsto \zeta_{t, 0}$ is a Poisson process with jump rate $\rho_{-}$.

To build up the random set $A_{t}$ we start with $\mathbb{N}_{0}^{2}$ such that $(0, j), 0 \leq j \leq \zeta_{+}$, and $(j, 0), 0 \leq$ $j \leq \zeta_{-}$are occupied. For completeness it is useful to regard also the sites $(j,-1),(-1, j)$, $j=0,1, \ldots$, as occupied. All remaining sites are empty. The set of occupied sites defines $A_{0}$. The site $(m, n)$ is filled after the random waiting time $w(m, n)$ starting from the instant of time when both the left neighbor at $(m-1, n)$ and the lower neighbor at $(m, n-1)$ are filled. $A_{t}$ is the set of occupied sites at time $t$. Its boundary, $\tilde{h}_{t}$, is defined in the coordinate system rotated by $\pi / 4$, to say the coordinates $(m, n)$ are transformed to the new coordinates $(j, h)$ through $j=m-n, h=m+n$. The distribution of $\tilde{h}_{t}$ agrees with $h_{t}$ inside the cone $\mathcal{C}=\{(j, h)|h \geq| j \mid\}$ : by construction the events at the boundary of $\mathcal{C}$ have the probability induced by the TASEP dynamics. In the interior of $\mathcal{C}$ the random variables $w(i, j)$ are the waiting times for the particle jumps of the TASEP.

Returning to the problem of the introduction, we have to set $\rho_{-}=\frac{1}{2}=\rho_{+}$. We define $t \mapsto \tilde{N}_{t}$ as the inverse function to $n \mapsto G(n, n)$. By Theorem 2.1 $\tilde{N}_{t}=N_{t}$ in distribution. Therefore, if one can control the statistics of the last passage time $G(n, n)$ for large $n$, one knows the statistics of the current across the origin for large $t$. More specifically, since $N_{t}$ and $G(n, n)$ are linear in average, their fluctuations have the same asymptotic distribution up to a linear change in scale.

\section{Defect lines}

There are other cases of interest which can be mapped to a last passage problem. As one example we choose the (deterministic) initial configuration as $\eta_{0, j}=\frac{1}{2}\left(1+(-1)^{j}\right)$. As before, we want to determine the statistics of $N_{t}-\frac{1}{4} t$ for large $t$. The last passage refers now to the upper triangle $\Delta_{n}=\{(i, j) \mid 1 \leq i, j \leq n, i+j \geq n+1\}$. Let $\Omega_{n}$ be the set of all up/right paths starting at the anti-diagonal $\{(i, j) \mid i+j=n+1\}$ and ending at $(n, n)$. Then

$$
G^{\mathrm{pl}}(n)=\max _{\omega \in \Omega_{n}} T(\omega) .
$$

$G^{\mathrm{pl}}(n)$ is the point to line last passage time, in contrast to $G(m, n)$ which is the point to point last passage time. As before $n \mapsto G^{\mathrm{pl}}(2 n)$ is the inverse function to $t \mapsto N_{t}$. Odd arguments would correspond to the initial condition $\eta_{0, j}=\frac{1}{2}\left(1-(-1)^{j}\right)$. 
One can force the alternating initial condition into the scheme of Section 2 . We set $\rho_{-}=1$, $\rho_{+}=0$, and reflect the $w(i, j)$ at the anti-diagonal, i.e. $w(i, j)=w(n+1-j, n+1-i)$ for $i+j \neq n+1$. On the anti-diagonal we set $w(i, j)=0$. Then in distribution $2 G^{\mathrm{pl}}(n)=G(n, n)$, where $G(n, n)$ is defined as in (2.1). In this scheme we have point to point last passage with a passage time distribution symmetric relative to the anti-diagonal.

A second example is the semi-infinite TASEP with a source at the origin. At site 1 we insert a particle with rate $\alpha, \alpha>0$, provided site 1 is empty. All other jumps are governed by the TASEP rule. The initial measure is Bernoulli $\rho$. Then the last passage is restricted to the lower triangle $\{(m, n) \mid n \leq m\}$. The right boundary of the last passage percolation is unchanged. The $w(i, j), j<i$, are exponential with mean 1 and the $w(j, j)$ are exponential with mean $\alpha$. The diagonal is a defect line. To make the formal analogy even closer to the previous cases we could copy the $w(i, j)$ in the lower triangle to the one in the upper triangle, which does not change the statistics of the last passage time from $(0,0)$ to $(n, n)$.

A widely studied case is the slow bond problem [8, 9]. As initial measure we take $\rho_{-}=1$, $\rho_{+}=0$. We assume that the jumps through the bond $(0,1)$ occur with rate $r$. This corresponds again to a defect line along the diagonal, where $w(j, j)$ is exponential with rate $r$. In contrast to the semi-infinite system the $w(i, j)$ 's for $i<j$ and for $i>j$ are now independent. One would like to know the average current for $t \rightarrow \infty, j_{\infty}(r)=\lim _{t \rightarrow \infty} N_{t} / t$. Clearly, $j_{\infty}(r)=r$ for small $r$ and $j_{\infty}(r)=\frac{1}{4}$ for $r \geq 1$. The critical rate $r_{c}$ is the smallest with $j_{\infty}\left(r_{c}\right)=\frac{1}{4}$. One knows that

$\frac{1}{2}<r_{c} \leq 1$ and conjectures $r_{c}=1$. In terms of the directed polymer, at $r_{c}$ there is a depinning transition. For small $r$ the directed polymer with end points $(1,1)$ and $(n, n)$ will stay order 1 close to the diagonal. As $r$ is increased, the size of excursions away from the diagonal increases. For $r>r_{c}$ the transverse fluctuations diverge as $n^{2 / 3}$ [10]. The directed polymer depins from the diagonal.

\section{The two-point function}

The TASEP with Bernoulli $\mu_{\rho}\left(\rho_{+}=\rho=\rho_{-}\right)$as starting measure is stationary in space-time. From a statistical mechanics point of view the central quantity is the two-point function

$$
S(j, t)=\mathbb{E}_{\rho}\left(\eta_{t, j} \eta_{0,0}\right)-\rho^{2}
$$

We list a few properties:

Proposition 4.1 We have $S(j, t) \geq 0, \sum_{j} S(j, t)=\rho(1-\rho), \sum_{j} j S(j, t)=\rho(1-\rho)(1-2 \rho) t$, and

$$
8 S(j, t)=\Delta\left(\mathbb{E}_{\rho}\left(h_{t}(j)^{2}\right)-\mathbb{E}_{\rho}\left(h_{t}(j)\right)^{2}\right)
$$

with the discrete Laplacian $(\Delta f)_{j}=f_{j+1}-2 f_{j}+f_{j-1}$ and $h_{t}(j)$ defined in (2.1). 
Proof: $S(j, t) / \rho(1-\rho)$ is the transition probability for a second class particle starting at the origin [11], which implies the first two assertions. For the third one we note that

$$
\begin{aligned}
\frac{d}{d t} \sum_{j} j S(j, t) & =\sum_{j} j \mathbb{E}_{\rho}\left(\left(\eta_{-t, 0}-\rho\right)\left(\eta_{0, j-1}\left(1-\eta_{0, j}\right)-\eta_{0, j}\left(1-\eta_{0, j+1}\right)\right)\right) \\
& =\sum_{j} \mathbb{E}_{\rho}\left(\left(\eta_{-t, j}-\rho\right) \eta_{0,0}\left(1-\eta_{0,1}\right)\right)=\sum_{j} \mathbb{E}_{\rho}\left(\left(\eta_{0, j}-\rho\right) \eta_{0,0}\left(1-\eta_{0,1}\right)\right) \\
& =\rho(1-\rho)(1-2 \rho),
\end{aligned}
$$

where we used conservation of the number of particles.

To see the fourth identity we compute

$$
\begin{aligned}
& \Delta \mathbb{E}_{\rho}\left(h_{t}(j)^{2}\right)=\mathbb{E}_{\rho}\left(h_{t}(j+1)^{2}-2 h_{t}(j)^{2}+h_{t}(j-1)^{2}\right) \\
& =-8 \mathbb{E}_{\rho}\left(N_{t}\left(\eta_{t, j+1}-\eta_{t, j}\right)\right)+2-4 \mathbb{E}_{\rho}\left(\left(1-2 \eta_{0, j}\right)\left(\eta_{0, j+1}-\eta_{0, j}\right)\right)
\end{aligned}
$$

using (2.1) and stationarity. By translation invariance

$$
\mathbb{E}_{\rho}\left(N_{t}\left(\eta_{t, j+1}-\eta_{t, j}\right)\right)=\mathbb{E}_{\rho}\left(\left(N_{t}^{-}-N_{t}\right) \eta_{t, j}\right)
$$

where $N_{t}^{-}$is the number of particles jumping through the bond $(-1,0)$ up to time $t$. The conservation law ensures that

$$
N_{t}^{-}+\eta_{0,0}=N_{t}+\eta_{t, 0}
$$

Inserting into 4.5$)$ yields

$$
\mathbb{E}_{\rho}\left(N_{t}\left(\eta_{t, j+1}-\eta_{t, j}\right)\right)=\mathbb{E}_{\rho}\left(\left(\eta_{t, 0}-\eta_{0,0}\right) \eta_{t, j}\right)
$$

and taking into account that $\mathbb{E}_{\rho}\left(h_{t}(j)\right)=2 \rho(1-\rho) t+(1-2 \rho) j$ we obtain (4.2).

$(\rho(1-\rho))^{-1} S(j, t)$ is a probability distribution with mean $(1-2 \rho) t$. One expects [12] that its variance grows as $t^{4 / 3}$ which suggests the scaling form

$$
S(j, t) \cong \rho(1-\rho)\left(4(\rho(1-\rho))^{1 / 3} t^{2 / 3}\right)^{-1} \frac{1}{8} g^{\prime \prime}\left((j-(1-2 \rho) t)\left(4(\rho(1-\rho))^{1 / 3} t^{2 / 3}\right)^{-1}\right) .
$$

The link to the last passage percolation is provided by (4.2) and Theorem 2.1, which tell us that the two-point function is given through the second moment of the last passage time at boundary conditions $\rho_{-}=\rho, \rho_{+}=\rho$. Because of the subtracted mean in (4.8) we consider the passage from $(0,0)$ to $(m, n)$ for large $n$ with fixed slope $\theta(\rho)=n / m=(1-\rho(1-\rho)-(1-$ $2 \rho)) /(1-\rho(1-\rho)+(1-2 \rho))$. The second moment of this last passage time is proportional to $g(0)$ with $g$ the scaling function in (4.8). To have the full function $g$ we have to determine the last passage time with an end point of order $n^{2 / 3}$ away from $(n / \theta(\rho), n)$. 


\section{The Baik and Rains analysis}

Recently Baik and Rains [5] studied last passage percolation with the above described boundary conditions. Unfortunately the case of an exponential distribution is not yet accessible to their techniques. Only the result of Johansson [2] is available which in our notation corresponds to $\rho_{-}=1, \rho_{+}=0$. For this case he proves that

$$
\lim _{t \rightarrow \infty} t^{-1 / 3} 2^{4 / 3}\left(N_{t}-\frac{1}{4} t\right)=-\xi_{\mathrm{GUE}}
$$

in distribution with $\mathbb{P}\left(\xi_{\mathrm{GUE}} \leq x\right)=F_{\mathrm{GUE}}(x)$. $F_{\mathrm{GUE}}$ is expressed in terms of a solution to the Painlevé II equation. We will consistently use the notation of [5] for the various distribution functions and will not repeat their definitions here.

Baik and Rains study the geometric and Poisson last passage percolation.

(i) geometric. The exponentially distributed random variables $w(i, j)$ are replaced by $w_{q}(i, j)$, $0<q<1$. $w_{q}(i, j), i, j \geq 1$, has geometric distribution with parameter $q, w_{q}(i, 0), i \geq 1$, has geometric distribution with parameter $\alpha_{+} \sqrt{q}$, and $w_{q}(0, j), j \geq 1$, has geometric distribution with parameter $\alpha_{-\sqrt{q}}$. The random variables $\left\{w_{q}(i, j) \mid i, j \geq 0\right\}$ are independent. Baik and Rains study the distribution of the passage time $G_{q}(n, n)$ for large $n$ in dependence on $q, \alpha_{+}$, $\alpha_{-}$, where they allow $\alpha_{+}, \alpha_{-}$being close to 1 . Specifically they set

$$
\alpha_{ \pm}=1-n^{-1 / 3} w_{ \pm}
$$

and establish how the asymptotic distribution of $G_{q}(n, n)$ depends on $w_{ \pm}$. The particular case $\alpha_{-}=0=\alpha_{+}$is proved by Johansson [2], who can allow for an arbitrary end point. In [13] last passage percolation is investigated for $\alpha_{-}=0=\alpha_{+}$and the $w_{q}(i, j)$ 's satisfying various reflection symmetries including the cases of interest here, reflection symmetric relative to the diagonal resp. relative to the anti-diagonal. The large $n$ distribution of $G_{q}(n, n)$ is established.

Following the scheme already explained we see that the geometric case can be interpreted as a discrete time TASEP with waiting times $w_{q}(i, j)+1$. In a single time step every particle which has an empty site ahead can jump to the right. They do so independently with probability $1-q$. Thus the initial step, left density $\rho_{-}$and right density $\rho_{+}$, is covered by [5] and the periodic initial configuration, ...0101 ..., by [13]. To handle the two-point function, a small change in the boundary density as in (5.2) should be translated to a small change in the angle of the end point for the directed polymer away from the diagonal. This seems to require a nontrivial generalization of the known results.

In the limit $q \rightarrow 1$ the random variables $(1-q) w_{q}(i, j)$ converge to $w(i, j)$ in distribution. Therefore the continuous time TASEP, time $t$, is recovered from the discrete time TASEP at integer times $[t /(1-q)]$ as $q \rightarrow 1$.

(ii) Poisson. We consider the geometric last passage percolation and interpret $w_{q}(i, j)$ as the number of points in the square with corners $\sqrt{q}\left(i \pm \frac{1}{2}, j \pm \frac{1}{2}\right)$. Then in the limit $q \rightarrow 0$ such 
that $(u, v)=\sqrt{q}(m, n)$ is fixed we obtain a Poisson point process which has unit density in the rectangle $[0, u] \times[0, v]$, line density $\alpha_{+}$on $[0, u] \times\{0\}$, and line density $\alpha_{-}$on $\{0\} \times[0, v]$. An allowed path, $\omega$, connects continuously $(0,0)$ to $(u, v)$, and is piecewise linear. Each linear segment has a slope $\theta$ such that $0 \leq \theta \leq \infty$ and connects two Poisson points, except for the first and last piece. $T(\omega)$ is the number of Poisson point which are transversed by $\omega$ and, as before,

$$
G_{\text {Poisson }}(u, v)=\max _{\omega} T(\omega) .
$$

For $\alpha_{-}=0=\alpha_{+}$the asymptotic distribution of $G_{\text {Poisson }}(v, v)$ is established in [14. Baik and Rains [5] extend to arbitrary $\alpha_{-}, \alpha_{+}$and allow for small deviations of the boundary densities as in (5.2) with $n^{-1 / 3}$ replaced by $v^{-1 / 3}$. Very recently they investigate the case of reflection symmetry relative to the diagonal, in particular $\alpha_{-}=\alpha_{+}$, and an extra line density along the diagonal [15].

The particle model behind the Poisson last passage percolation is the PNG model [16, 17, 18]. It consists of point particles with velocity \pm 1 . They annihilate each other at a collision and are created in \pm pairs with rate 1 . If $\rho_{+}(x, t)$ is the density of right movers, and $\rho_{-}(x, t)$ of left movers, then the field $\phi(x, t)=\rho_{+}(x, t)-\rho_{-}(x, t)$ is locally conserved and corresponds to $\eta_{t, j}$ of the TASEP. More precisely the corresponding height function

$$
h(x, t)=N_{t}-\int_{0}^{x} d y \phi(y, t)
$$

where as before $N_{t}$ is the time integrated current at the origin, $N_{t}=\int_{0}^{t} d s\left(\rho_{+}(0, s)+\rho_{-}(0, s)\right)$. The height is related to the last passage time through $G(u, v)=h(x, t)$ in distribution with $u=(t+x) / \sqrt{2}, v=(t-x) / \sqrt{2}$.

In contrast to the geometric and exponential last passage percolation, a change in the boundary densities $\alpha_{ \pm}$can be compensated by a variation of the end point. For example, for the square $[0, v]^{2}$ with boundary densities $\alpha_{ \pm}=\left(1-v^{-1 / 3} w\right)^{ \pm 1}$, we can stretch the 1 -axis by $1 / \alpha_{+}$and the 2 -axis by $1 / \alpha_{-}$. Then the boundary densities are 1 and the end point of the polymer is tilted by a distance $v^{2 / 3}$ away from $(v, v)$, which is the quantity needed for the scaling form of the 2-point function. Thus for the PNG model the scaling form of $\mathbb{E}(\phi(x, t) \phi(0,0))$ follows from the analogue Proposition 4.1 for PNG and the asymptotic analysis in [5], compare with Section 7.2.

The defect line along the diagonal is most easily visualized in the growth version of PNG, where unit up-steps are particles with velocity -1 and unit down-steps with velocity 1 . If $\alpha_{-}=0=\alpha_{+}$, nucleation of pairs of steps are allowed only above the ground layer $[-t, t]$. Extra Poisson points along the diagonal with line density $\alpha$ correspond to a source at the origin which nucleates at rate $\alpha$. The full PNG droplet has no reflection symmetry at the diagonal. With reflection symmetry one restricts oneself to the half droplet, where nucleations only above the ground layer $[0, t]$ are allowed. In the depinned phase the extra mass is incorporated in the 
droplet without visible modification of the macroscopic shape. In the pinned phase an extra pile of linear slope is created on top of the PNG droplet.

\section{Scaling theory}

In limit theorems one has to separate universal from model-dependent features. To give the standard example: let $\xi_{j}, j \in \mathbb{Z}$, be a stationary mean zero sequence of random variables which satisfies the central limit theorem as

$$
\lim _{n \rightarrow \infty} \mathbb{P}\left(\frac{1}{\sqrt{n}} \sum_{j=1}^{n} \xi_{j} \leq \sigma x\right)=F_{\mathrm{G}}(x) .
$$

Here $F_{\mathrm{G}}$ is the distribution function of a standard Gaussian random variable. $F_{\mathrm{G}}$ is universal (within well-studied limits), whereas the variance $\sigma^{2}$ depends on the particular probability law for the $\xi_{j}$ 's. In our example $\sigma^{2}$ is easily computed as

$$
\sigma^{2}=\sum_{j=-\infty}^{\infty} \mathbb{E}\left(\xi_{j} \xi_{0}\right)
$$

For the ASEP and similar growth models a corresponding scaling theory is available which determines the model-dependent longitudinal and transverse scales [19. To apply the theory one has to know the stationary measures as labeled by their mean density, $\rho$. The two basic quantities are

$$
\begin{aligned}
& j(\rho)=\text { average current at density } \rho \\
& A(\rho)=\text { size of the density fluctuations in the stationary measure. }
\end{aligned}
$$

If the density is regarded as the slope of a height function as in (2.1), then $A(\rho)$ is the roughness amplitude for $j \mapsto h(j)$ in the stationary measure, i.e. $A(\rho)=\lim _{j \rightarrow \infty} j^{-1} \mathbb{E}([h(j)-h(0)-$ $\left.\mathbb{E}(h(j)-h(0))]^{2}\right)$.

For the TASEP $j(\rho)=\mu_{\rho}\left(\eta_{0}\left(1-\eta_{1}\right)\right)=\rho(1-\rho)$ and the height diffusion constant

$$
A(\rho)=\lim _{j \rightarrow \infty} \frac{1}{j} \mu_{\rho}\left((h(j)-(1-2 \rho) j)^{2}\right)=4 \sum_{j}\left(\mu_{\rho}\left(\eta_{j} \eta_{0}\right)-\rho^{2}\right)=4 \rho(1-\rho) .
$$

For the discrete time TASEP, jump probability $1-q$, the stationary measure at density $\rho$ is a Markov chain with transition probability $Q$, a $2 \times 2$-matrix which we write in the form $Q_{00}=1-r, Q_{01}=r, Q_{10}=s, Q_{11}=1-s . r, s$ are determined through $(1-r)(1-s)=$ qrs and $(r+s) \rho=s$. Then the average current

$$
j(\rho)=\rho s
$$

and the roughness amplitude

$$
A(\rho)=4 \sum_{j=-\infty}^{\infty}\left(\mathbb{E}_{\rho}\left(\eta_{j} \eta_{0}\right)-\rho^{2}\right)=4\left(\rho(1-\rho)+2 \sum_{j=1}^{\infty}\left(\rho\left(Q^{j}\right)_{11}-\rho^{2}\right)\right) .
$$


For the PNG model in the stationary measure the + , resp. - , particles are Poisson distributed with density $\rho_{+}$, resp. $\rho_{-}$, satisfying the stationarity constraint $2 \rho_{+} \rho_{-}=1$. The conserved density is $\rho=\rho_{+}-\rho_{-}$and the current

$$
j(\rho)=\rho_{+}+\rho_{-}=\left(2+\rho^{2}\right)^{1 / 2} .
$$

From the Poisson property we conclude

$$
A(\rho)=\rho_{+}+\rho_{-}=\left(2+\rho^{2}\right)^{1 / 2} .
$$

$j^{\prime}(\rho)=v(\rho)$ is the velocity of the density fluctuations and $\lambda=j^{\prime \prime}(\rho)$ is the KPZ coupling constant [19]. In terms of these quantities, the scale in the $h$-direction is

$$
\operatorname{sign} \lambda\left(\frac{1}{2}|\lambda| A^{2}\right)^{1 / 3} t^{1 / 3}
$$

and the scale in the transverse $j$-direction is

$$
2^{4 / 3}\left(\lambda^{2} A\right)^{1 / 3} t^{2 / 3}
$$

For the TASEP, since $\lambda=-1$, the $h$-direction then comes in units of $-2^{-1 / 3}(4 \rho(1-\rho))^{2 / 3} t^{1 / 3}$, whereas the $j$-direction comes in units of $4(\rho(1-\rho))^{1 / 3} t^{2 / 3}$.

In (6.8) and (6.9) we have fixed two dimensionless scale factors. Their role is most easily explained in our entry example, where we could have adopted a definition of the error function $F_{\mathrm{G}}$ such that the Gaussian has mean $1 / 2$. Then in (6.1) $\sigma x$ is to be replaced by $\sqrt{2} \sigma x$ independently of the value of $\sigma$. The particular convention is determined through the comparison with one single test case. In our context we follow [5] in the definition of the distribution functions, who rely on the established conventions for the Painlevé II Riemann-Hilbert problem. The test case ist the PNG model, which fixes the prefactors in (6.8) to $2^{-1 / 3}$ and in (6.9) to $2^{4 / 3}$.

So far proofs are available only for a short list of models. In all cases the model-dependent parameters are such as to agree with the scaling theory. In particular the discrete time TASEP provides a two-parameter $(\rho$ and $q$ ) test of the theory.

\section{Current fluctuations}

There is little doubt that the results of Baik and Rains also hold in the limit of an exponential distribution when $q \rightarrow 1$. Strictly speaking our results are conjectures, except for $\rho_{-}=1$, $\rho_{+}=0$ [2]. We discuss the list of our examples. Some of them have been announced in [18], where also numerical plots of $F_{\mathrm{GUE}}, F_{\mathrm{GOE}}$, and $F_{0}$ are given.

\subsection{Initial step density}

Fluctuation results for $N_{t}$ are most easily summarized in the diagram of Figure 11. We first remind the reader of the limit measure $\mu_{\infty}$ for $\left\{\eta_{t, j},|j| \leq N\right\}, N$ arbitrary, $t \rightarrow \infty$ [6]. The 


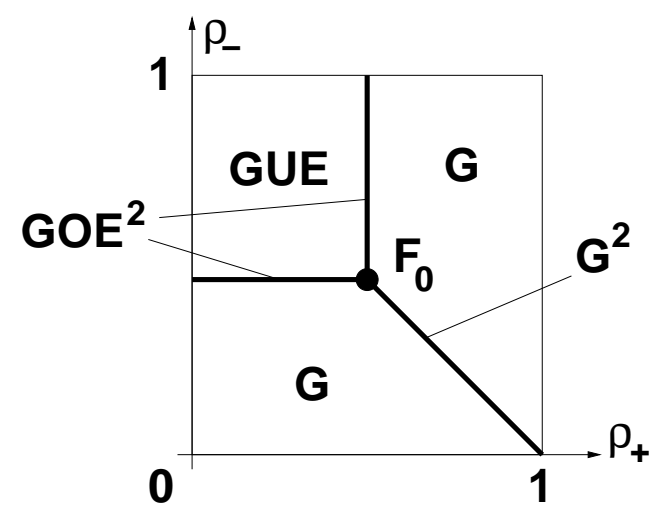

Figure 1: Fluctuation phase diagram for the TASEP.

upper left corner is the maximal current phase with $\mu_{\infty}=\mu_{1 / 2}$. In the lower left $\mu_{\infty}=\mu_{\rho_{-}}$and in the upper right $\mu_{\infty}=\mu_{\rho_{+}}$. Along the line $\rho_{-}+\rho_{+}=1$ the long time limit is the superposition $\mu_{\infty}=\frac{1}{2} \mu_{\rho_{-}}+\frac{1}{2} \mu_{\rho_{+}}$. In particular, we have $\mathbb{E}\left(N_{t}\right) / t \rightarrow \mu_{\infty}\left(\eta_{0}\left(1-\eta_{1}\right)\right)$ as $t \rightarrow \infty$.

To understand the fluctuations of $N_{t}$ we use the directed polymer picture and recall that the polymer starts at $(0,0)$ and ends at $(n, n)$. If $\rho_{-}<1-\rho_{+}, \rho_{-}<\frac{1}{2}$, then the polymer stays a finite fraction of steps at the right edge. Since for the edge steps the passage time is a sum of independent exponentials, its fluctuations are of order $\sqrt{t}$ and Gaussian (G). At some point the polymer must enter the bulk and we expect subleading corrections of order $t^{1 / 3}$,

$$
N_{t} \cong \rho(1-\rho) t+t^{1 / 2} \xi_{\mathrm{G}}-t^{1 / 3} \xi_{\mathrm{GUE}}
$$

up to prefactors, with either $\rho=\rho_{-}$or $\rho=\rho_{+}$. If $\rho_{-}+\rho_{+}=1, \rho_{-}<\frac{1}{2}$, then the polymer has a choice between the left and right edge and the fluctuations of $N_{t}$ are the maximum of two independent Gaussians $\left(\mathrm{G}^{2}\right)$. Recall that if $\xi_{i}$ has distribution function $F_{i}, i=1,2$, then their maximum has the distribution function $F_{1} F_{2}$ provided $\xi_{1}, \xi_{2}$ are independent, hence our notation.

If $\rho_{-}>\frac{1}{2}, \rho_{+}<\frac{1}{2}$, then it does not pay for the polymer to stay at neither edge. The fluctuations must be as for the Johansson case of trivial edges, $\rho_{-}=1, \rho_{+}=0$, which have $F_{\text {GUE }}$ as distribution function. Thus the difficult cases are the two critical lines $\rho_{-}=\frac{1}{2}, \rho_{+}<\frac{1}{2}$, resp. $\rho_{-}>\frac{1}{2}, \rho_{+}=\frac{1}{2}$, and the critical point $\rho_{-}=\frac{1}{2}=\rho_{+}$, which corresponds to the Bernoulli $\frac{1}{2}$ initial measure. On the line $\rho_{-}=\frac{1}{2}\left(\rho_{+}=\frac{1}{2}\right)$ the directed polymer stays order $n^{1 / 3}$ at the left (right) edge, whereas at the critical point it makes a choice between the two edges. According to [5] the fluctuations of $N_{t}$ are of order $t^{1 / 3}$ and are GOE ${ }^{2}$ distributed on the critical lines, $F_{0}$ distributed at the critical point.

From the point of view of the last passage percolation the end point $(n, n)$ is somewhat special and one might consider more generally the end point $(m, n)$ with the slope $\theta=n / m$ fixed. To have a firm link to the TASEP we will use the parameterization (2.1), in which case the quantity of interest is $h_{t}([y t]),|y|<1$, [.] denoting the integer part. $h_{t}(0)=2 N_{t}$ is the 
particular case just explained. The hydrodynamic theory [20, 1] establishes the law of large numbers for $h_{t}([y t])$ with the result

$$
\lim _{t \rightarrow \infty} \frac{1}{t} h_{t}([y t])=\bar{h}(y)
$$

almost surely for $|y|<1$. The limit $\bar{h}$ depends on $\rho_{-}, \rho_{+}$and is given as follows: If $\rho_{-}<\rho_{+}$, then

$$
\bar{h}(y)= \begin{cases}\left(1-2 \rho_{-}\right) y+2 \rho_{-}\left(1-\rho_{-}\right) & \text {for } \quad y \leq y_{c} \\ \left(1-2 \rho_{+}\right) y+2 \rho_{+}\left(1-\rho_{+}\right) & \text {for } \quad y>y_{c}\end{cases}
$$

with $y_{c}=\left(\rho_{+}\left(1-\rho_{+}\right)-\rho_{-}\left(1-\rho_{-}\right)\right) /\left(\rho_{+}-\rho_{-}\right)$. If $\rho_{-}>\rho_{+}$, then

$$
\bar{h}(y)=\left\{\begin{array}{llc}
\left(1-2 \rho_{-}\right) y+2 \rho_{-}\left(1-\rho_{-}\right) & \text {for } & y \leq 1-2 \rho_{-} \\
\frac{1}{2}\left(y^{2}+1\right) & \text { for } & 1-2 \rho_{-}<y \leq 1-2 \rho_{+} \\
\left(1-2 \rho_{+}\right) y+2 \rho_{+}\left(1-\rho_{+}\right) & \text {for } & 1-2 \rho_{+}<y
\end{array}\right.
$$

We are interested in the fluctuations of

$$
h_{t}([y t])-t \bar{h}(y)
$$

for fixed $y$ in the limit $t \rightarrow \infty$. If $\bar{h}$ is linear around $y$, then the fluctuations are order $\sqrt{t}$ and Gaussian. If $\bar{h}$ has a cusp at $y$ (and is linear on both sides), then the fluctuations are order $\sqrt{t}$ and $\mathrm{G}^{2}$. On the other hand, if $\bar{h}$ has nonzero curvature at $y$ then the fluctuations are order $t^{1 / 3}$ and GUE. The critical lines, $\mathrm{GOE}^{2}$, correspond to a $y$ where at $\bar{h}(y)$ the curved piece joins the linear piece. Finally the critical point, $F_{0}$, is the merger of the two critical lines.

We summarize our findings in the form of a (well-founded) conjecture. $\mathbb{P}_{\rho_{-}, \rho_{+}}$refers to the TASEP with $\mu_{\rho_{-}, \rho_{+}}$as starting measure. $h_{t}(j)$ is defined in (2.1), $\bar{h}$ in (7.3), (7.4).

Conjecture $7.1(\mathrm{G})$ Let either $\rho_{-}<\rho_{+}$and $y>y_{c}$ or $\rho_{-}>\rho_{+}$and $y>1-2 \rho_{+}$. Then

$$
\lim _{t \rightarrow \infty} \mathbb{P}_{\rho_{-}, \rho_{+}}\left(t \bar{h}(y)-h_{t}([y t]) \leq\left(4 \rho_{+}\left(1-\rho_{+}\right)\left(y-1+2 \rho_{+}\right) t\right)^{1 / 2} x\right)=F_{\mathrm{G}}(x)
$$

Let either $\rho_{-}<\rho_{+}$and $y<y_{c}$ or $\rho_{-}>\rho_{+}$and $y<1-2 \rho_{-}$. Then

$$
\lim _{t \rightarrow \infty} \mathbb{P}_{\rho_{-}, \rho_{+}}\left(t \bar{h}(y)-h_{t}([y t]) \leq\left(4 \rho_{-}\left(1-\rho_{-}\right)\left(-y+1-2 \rho_{-}\right) t\right)^{1 / 2} x\right)=F_{\mathrm{G}}(x) .
$$

$\left(\mathrm{G}^{2}\right)$ Let $\rho_{-}<\rho_{+}$and $y=y_{c}$, then

$$
\begin{aligned}
\lim _{t \rightarrow \infty} \mathbb{P}_{\rho_{-}, \rho_{+}}(t & \left.\bar{h}(y)-h_{t}([y t]) \leq\left(\left(\rho_{+}-\rho_{-}\right) t\right)^{1 / 2} x\right) \\
& =F_{\mathrm{G}}\left(\left(4 \rho_{+}\left(1-\rho_{+}\right)\right)^{-1 / 2} x\right) F_{\mathrm{G}}\left(\left(4 \rho_{-}\left(1-\rho_{-}\right)\right)^{-1 / 2} x\right) .
\end{aligned}
$$

(GUE) Let $\rho_{-}>\rho_{+}$and $1-2 \rho_{-}<y<1-2 \rho_{+}$. Then

$$
\lim _{t \rightarrow \infty} \mathbb{P}_{\rho_{-}, \rho_{+}}\left(t \bar{h}(y)-h_{t}([y t]) \leq 2^{-1 / 3}\left(1-y^{2}\right)^{2 / 3} t^{1 / 3} x\right)=F_{\mathrm{GUE}}(x) .
$$


$\left(\mathrm{GOE}^{2}\right)$ Let $\rho_{-}>\rho_{+}$and either $y=1-2 \rho_{-}$or $y=1-2 \rho_{+}$. Then

$$
\lim _{t \rightarrow \infty} \mathbb{P}_{\rho_{-}, \rho_{+}}\left(t \bar{h}(y)-h_{t}([y t]) \leq 2^{-1 / 3}\left(1-y^{2}\right)^{2 / 3} t^{1 / 3} x\right)=F_{\mathrm{GOE}}(x)^{2} .
$$

$\left(\mathrm{F}_{0}\right)$ Let $\rho_{-}=\rho=\rho_{+}$and $y=1-2 \rho$. Then

$$
\lim _{t \rightarrow \infty} \mathbb{P}_{\rho_{-}, \rho_{+}}\left(t \bar{h}(y)-h_{t}([y t]) \leq 2^{-1 / 3}\left(1-y^{2}\right)^{2 / 3} t^{1 / 3} x\right)=F_{0}(x) .
$$

$F_{\mathrm{G}}$ is the distribution function of a standard normal distributed random variable. $F_{0}$ and the Tracy-Widom distribution functions $F_{\mathrm{GUE}}, F_{\mathrm{GOE}}$ are defined in [5].

The Gaussian case $(\mathrm{G})$ with $\rho_{-}=\rho_{+}$is proved in [21] and the case (GUE) with $\rho_{-}=1, \rho_{+}=0$ in [2].

In (7.6) to (7.11) the random variable $-\left(h_{t}([y t])-t \bar{h}(y)\right)$ appears because of the inversion from the passage time $G(m, n)$ to the height. In each case we have subtracted the asymptotic mean as computed from the hydrodynamic theory. For the variances of the Gaussians we have to determine how many steps, on the scale $n$, the directed polymer stays at either edge. For the prefactor $2^{-1 / 3}\left(1-y^{2}\right)^{2 / 3}$ in (7.9) to (7.11) we either take the limit of the discrete time TASEP or use the scaling theory of Section 6. Because of the inversion, also the lower and upper tails are interchanged. For example $F_{\mathrm{GUE}}(x)$ has the lower tail $\exp \left(-|x|^{3} / 12\right)$ and the upper tail $\exp \left(-4|x|^{3 / 2} / 3\right)$.

\subsection{Stationary two-point function}

The starting measure is Bernoulli $\rho$ and $\mathbb{P}_{\rho}$ is the corresponding path measure of the stationary TASEP, $0<\rho<1$. Density fluctuations propagate with velocity $1-2 \rho$. Therefore the anomalous fluctuations appear in $h_{t}([(1-2 \rho) t])$, compare with (7.11). For the full two-point function we need the height distribution a distance of order $t^{2 / 3}$ away, i.e. at $(1-2 \rho) t+4(\rho(1-$ $\rho))^{1 / 3} t^{2 / 3} w$ for arbitrary $w$, compare with (6.9).

Conjecture 7.2 We have

$$
\begin{aligned}
\lim _{t \rightarrow \infty} \mathbb{P}_{\rho}( & -h_{t}\left(\left[(1-2 \rho) t+4(\rho(1-\rho))^{1 / 3} t^{2 / 3} w\right]\right)+2 \rho(1-\rho) t \\
& \left.\quad+(1-2 \rho)\left((1-2 \rho) t+4(\rho(1-\rho))^{1 / 3} t^{2 / 3} w\right) \leq 2(\rho(1-\rho))^{2 / 3} t^{1 / 3} x\right) \\
= & F_{w}(x) .
\end{aligned}
$$

The distribution function $F_{w}(x)$ is defined in [5] and denoted there by $H\left(4 w^{2}+x ; w,-w\right)$. The distribution function $F_{0}$ appears also in (7.11).

Note that $\int d F_{w}(x) x=0$, since we have subtracted the average of $h_{t}$. The prefactors of $x$ and $w$ are infered from the scaling theory of Section 6. For the PNG model, the analogue of (7.12) 


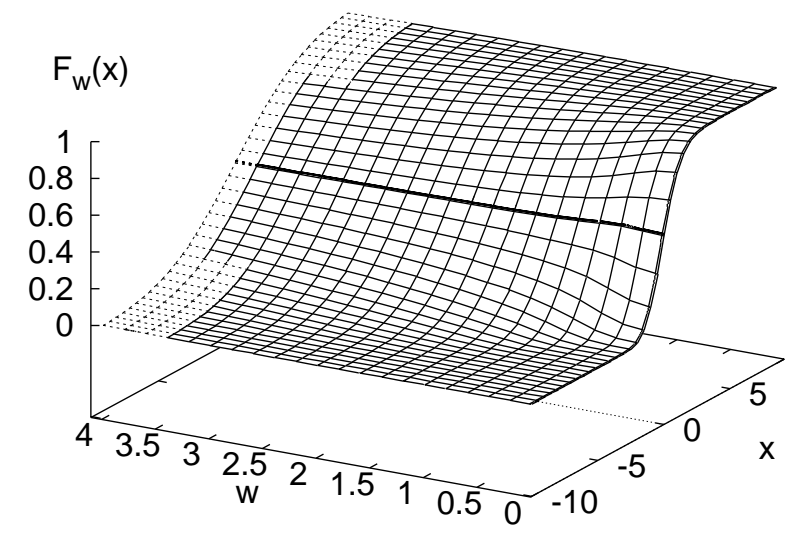

Figure 2: A 3d-plot of the distribution functions $F_{w}$ for $w>0$.

follows from [5]. If the convergence (7.12) holds also for the second moments, then

$$
\begin{aligned}
& \mathbb{E}_{\rho}\left(\left[h_{t}\left([(1-2 \rho)) t+4(\rho(1-\rho))^{1 / 3} t^{2 / 3} w\right]\right)-2 \rho(1-\rho) t\right. \\
& \left.\left.-(1-2 \rho)\left((1-2 \rho) t+4(\rho(1-\rho))^{1 / 3} t^{2 / 3} w\right)\right]^{2}\right) \\
& \cong 4(\rho(1-\rho))^{4 / 3} t^{2 / 3} \int d F_{w}(x) x^{2}
\end{aligned}
$$

for large $t$. Setting

$$
g(w)=\int d F_{w}(x) x^{2}
$$

and taking second derivatives in (7.13), we conclude the scaling form of (4.8).

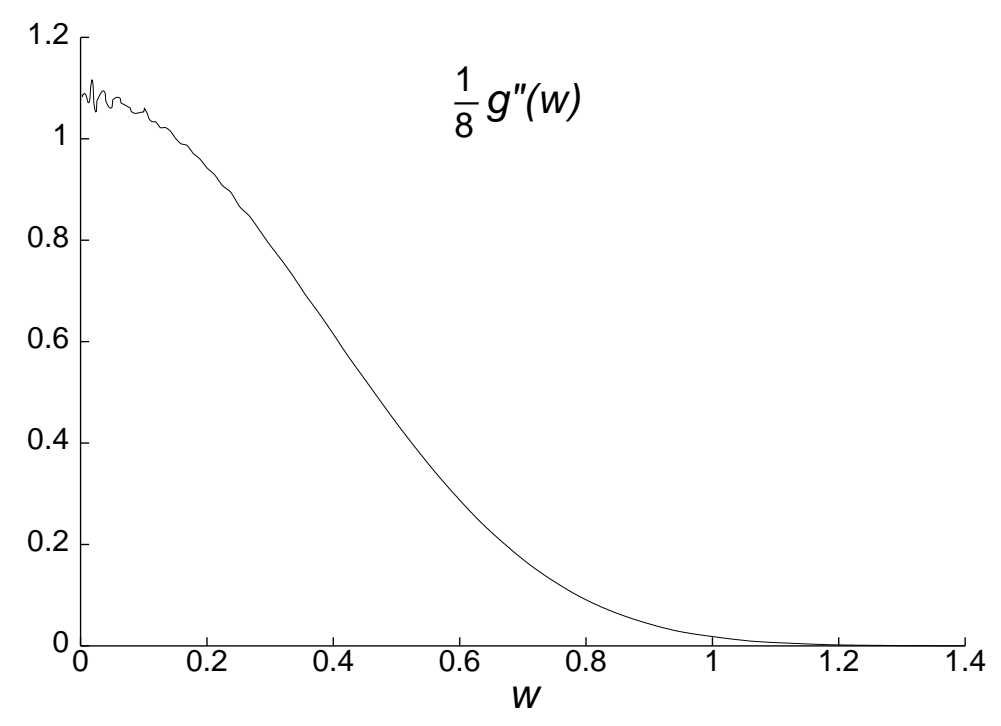

Figure 3: The second derivative of the second moment of $F_{w}$ vs. $w$.

$F_{w}(x)$ is the solution of a first order partial differential equation with suitable boundary conditions. A numerical plot is provided in Figure 2. In essence it shows how the distribution 
broadens for $w \rightarrow \infty$, reflecting the cross over to the Gaussian fluctuations as one moves away from the characteristic $\{j=(1-2 \rho) t\}$. The second moment, $g(w)$, is symmetric and increases linearly as $4|w|$ for large $|w|$. In Figure 3 we plot the scaling function $g^{\prime \prime}$ as determined from the numerical evaluation of the second moment of $F_{w}$ at various values of $w$. The oscillations at small $w$ result from numerical imprecisions for $F_{w}$. In [12] an approximate nonlinear integral equation for $g^{\prime \prime}$ has been derived, which was then solved numerically in [22]. The approximate $g(0)$ differs from the exact one by an order of $5 \%$. From the available data a more accurate comparison does not seem to be feasible.

\subsection{Semi-infinite system}

We restrict to the half-lattice $\mathbb{N}$ and insert with rate $\alpha$ particles at site 1 , respecting the constraint $\eta_{1}=0,1$. The initial measure is Bernoulli $\mu_{\rho}$. The last passage percolation representation lives in the lower triangle $\{(i, j) \mid 0 \leq j \leq i\}$. Along the diagonal the $w(j, j)$ are exponential with rate $\alpha$ and at the lower edge the $w(j, 0)$ are exponential with rate $1-\rho$. By maximizing the passage time on scale $n$ we obtain the same phase diagram as for the critical step. However the fluctuations in $N_{t}$, now the number of particles injected up to time $t$, are modified. In Figure 4 we summarize the findings [15] which could be written more formally as in Conjecture 7.1 and so far have been proven only for the Poisson case, i.e. the semi-infinite PNG model with a source at the origin. GSE is the distribution of the largest eigenvalue of a symplectic Gaussian random matrix. $F_{0}^{\text {semi }}$ is a novel distribution and given by

$$
F_{0}^{\mathrm{semi}}(x)=\left(1+\frac{1}{2}(-v(x)+u(x))\left(x+2 u^{\prime}(x)+2 u(x)^{2}\right)\right) F(x) E(x)^{3}
$$

in the notation of [5]. On the anti-diagonal a new feature appears. Recall that for the infinite system the distribution $\mathrm{G}^{2}$ arose from the choice between the left and right edge. Here the directed polymer may stay for an arbitrary length at the lower edge and then switch to the diagonal. Optimizing over this one parameter family of paths yields for the passage time a

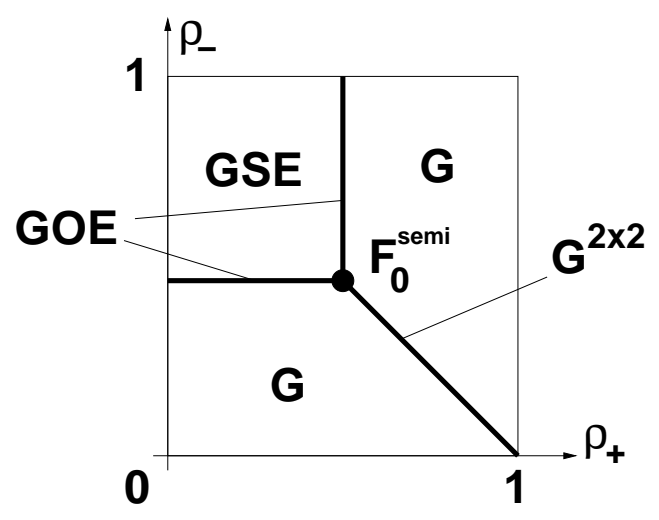

Figure 4: Fluctuation phase diagram for the semi-infinite TASEP. 
distribution which is given by the largest eigen value of a $2 \times 2$ GUE matrix, denoted by $\mathrm{G}^{2 \times 2}$ in Figure 4 .

\subsection{Defect lines, depinning}

For a change let us consider the Poisson last passage percolation. As already explained, in the square $[0, v]^{2}$ we have Poisson points with density 1 . The directed polymer starts at $(0,0)$ and ends at $(v, v)$. We add extra Poisson points along the diagonal with line density $\alpha$. Of primary interest is the last passage time, $\lim _{v \rightarrow \infty} v^{-1} G_{\alpha}(v)=\tau(\alpha)$. We know that $\tau(0)=2$. The critical $\alpha_{c}$ is defined as the largest $\alpha$ such that $\tau(\alpha)=2$. If $\alpha>\alpha_{c}$, the polymer is pinned and typically returns after a length $\ell_{\|}(\alpha)$ to the diagonal. Between two returns the polymer makes an excursion of size $\ell_{\perp}(\alpha)$ away from the diagonal. The depinning transition is governed by $\tau(\alpha), \ell_{\|}(\alpha), \ell_{\perp}(\alpha)$ as $\alpha \downarrow \alpha_{c}$. If $\ell_{\perp}\left(\alpha_{c}\right)<\infty$, the transition is first order, while $\ell_{\perp}(\alpha) \rightarrow \infty$ as $\alpha \downarrow \alpha_{c}$ signals as second order transition.

Since there is considerable interest from the physics of disordered systems, a phenomenological theory has been developed, which after some controversy seems to be widely accepted 23, 24]. We explain the predictions and first recall the pure problem with the path measure

$$
\left(Z_{t}\right)^{-1} \mathbb{P}^{B B} \exp \left[-\beta \int_{0}^{t} d s V\left(x_{s}\right)\right] .
$$

Here $x_{s} \in \mathbb{R}^{d}$ is a Brownian path which has the a priori weight given by the Brownian bridge $\mathbb{P}^{B B}$ with start and end point $0 . V$ is a pinning potential, which by a suitable choice of units is simply $V(x)=0$ for $|x| \geq 1$ and $V(x)=-1$ for $|x|<1$. $Z_{t}$ is the normalizing partition function. The analogue of $\tau(\alpha)$ is the ground state energy $e(\beta)=\lim _{t \rightarrow \infty}-t^{-1} \log Z_{t}, e(0)=0$. For $d=3$ a certain minimal strength is required to pin the Brownian motion. Thus $\beta_{c}>0$ and $e(\beta) \simeq-\left(\beta-\beta_{c}\right)^{2}$ for $\beta \geq \beta_{c}$. However for $d=2$, Brownian motion is null-recurrent and $\beta_{c}=0$. Just a tiny bit of attraction suffices to pin the polymer. Correspondingly $e(\beta)$ has an essential singularity as $e(\beta) \simeq-e^{-1 / \beta}, \ell_{\|}(\beta) \simeq e^{1 / \beta}, \ell_{\perp}(\beta) \simeq \ell_{\|}(\beta)^{1 / 2}$ for small $\beta$.

The claim is that a directed polymer in $1+1$ dimensions in a random potential is pinned in essence as a directed polymer in $2+1$ dimensions with zero bulk potential. Specifically, any attraction along the diagonal will pin the polymer, i.e. $\alpha_{c}=0$, the passage time $\tau(\alpha) \simeq 2+e^{-1 / \alpha}$, and the longitidonal excursions $\ell_{\|}(\alpha) \simeq e^{1 / \alpha}$ with the usual link $\ell_{\perp}(\alpha) \simeq \ell_{\|}(\alpha)^{2 / 3}$ for small $\alpha$. No rigorous result seems to be available.

Transcribed to the slow bond problem, the prediction is $r_{c}=1$ with an essential singularity for $j_{\infty}(r)$ at $r=1$.

To model the case where the Poisson points are reflection symmetric relative to the diagonal, we add in (7.16) a hard wall and replace $V$ by $V_{\mathrm{hw}}, V_{\mathrm{hw}}\left(x_{1}, x_{\perp}\right)=V(x)$ for $x_{1} \geq 0, V_{\mathrm{hw}}(x)=\infty$ for $x_{1}<0$. The entropic repulsion shifts to $\beta_{c}>0$ even for $d=1,2$. Therefore the prediction of the phenomenological theory is $\tau(\alpha) \simeq 2+c\left(\alpha-\alpha_{c}\right)^{2}, \ell_{\|}(\alpha) \simeq\left(\alpha-\alpha_{c}\right)^{-2}$, and $\ell_{\perp}(\alpha) \simeq$ $\ell_{\|}(\alpha)^{2 / 3}$ for $\alpha$ close to $\alpha_{c}, \alpha \geq \alpha_{c}$. This is in agreement with the exact result $\alpha_{c}=1 / \sqrt{2}$ and 
$\tau(\alpha)=2$ for $0 \leq \alpha \leq \alpha_{c}, \tau(\alpha)=\sqrt{2}(\alpha+(1 / 2 \alpha))$ for $\alpha_{c} \geq \alpha$, which slightly above $\alpha_{c}$ yields $\tau(\alpha) \simeq 2+2\left(\alpha-\alpha_{c}\right)^{2}$ [13.

\section{Conclusions}

It is rather surprising to have now, after more than 20 years of study, a technique available through which universal scaling functions can be computed, in some cases even very explicitly. The main lesson to be drawn is that the universal distribution functions on the scale $t^{1 / 3}$ depend on the type of initial conditions. It would be of interest to understand whether our list is already complete.

Despite progress there are several obvious elements missing. Firstly for the transverse fluctuations of the polymer we do not have such a fine information as on the passage time $(=$ energy of the polymer) [10]. More importantly, only single distributions, like the passage time with given end points, can be handled. To have a more detailed understanding of the energy landscape joint distributions must be studied, like the joint distribution of the first passage times $G\left(m_{1}, n_{1}\right), G\left(m_{2}, n_{2}\right)$ refering to two distinct end points.

\section{References}

[1] T. Seppäläinen, Hydrodynamic scaling, convex duality, and asymptotic shapes of growth models. Markov Processes Rel. Fields 4, 1-26 (1998)

[2] K. Johansson, Shape fluctuations and random matrices. Comm. Math. Phys. 209, 437-476 (2000)

[3] T. Seppäläinen, Large deviations for increasing sequences on the plane. Prob. Th. Rel. Fields 112, 221-244 (1998)

[4] J.-D. Deuschel, O. Zeitouni, On increasing subsequences of i.i.d. samples. Combinatorics, Probability and Computing 8, 247-263 (1999)

[5] J. Baik, E.M. Rains, Limiting distributions for a polynuclear growth model with external sources. J. Stat. Phys. 100, 523-542 (2000)

[6] T. M. Liggett, Ergodic theorems for the asymmetric simple exclusion process. Trans. Amer. Math. Soc. 213, 237-261 (1975)

[7] F. P. Kelly, Reversibility and Stochastic Networks. John Wiley \& Sons, Chichester (1979)

[8] S. A. Janowsky, J. L. Lebowitz, Exact results for the asymmetric simple exclusion process with a blockage. J. Stat. Phys. 77, 35-51 (1994) 
[9] T. Seppäläinen, Hydrodynamic profiles for the totally asymmetric exclusion process with a slow bond. J. Stat. Phys., to appear

[10] K. Johansson, Transversal fluctuations for increasing subsequences on the plane. Preprint, math.PR/9910146

[11] P. Ferrari, Shock fluctuations in the asymmetric simple exclusion. Prob. Th. Rel. Fields 91, 25-64 (1992)

[12] H. van Beijeren, R. Kutner, H. Spohn, Excess noise for driven diffusive systems. Phys. Rev. Lett. 54, 2026-2029 (1985)

[13] J. Baik, E.M. Rains, Symmetrized random permutations. Preprint, math.CO/9910019

[14] J. Baik, P.A. Deift, K. Johansson, On the distribution of the length of the longest increasing subsequence in a random permutation. J. Amer. Math. Soc. 12, 1119-1178 (1999)

[15] J. Baik, E.M. Rains, private communication

[16] J. Krug, H. Spohn, Anomalous fluctuations in the driven and damped sine-Gordon chain. Eur. Phys. Lett. 8, 219-224 (1989)

[17] M. Prähofer, H. Spohn, Statistical self-similarity of one-dimensional growth processes. Physica A279, 342-352 (2000)

[18] M. Prähofer, H. Spohn, Universal distribution for growth processes in $1+1$ dimensions and random matrices. Phys. Rev. Lett. 84, 4882-4885 (2000)

[19] J. Krug, P. Meakin, T. Halpin-Healy, Amplitude universality for driven interfaces and directed polymers in random media. Phys. Rev. A 45, 638-653 (1992)

[20] H. Rost, Non-equilibrium behavior of a many particle process: density profile and local equilibrium. Z. Wahrsch. Verw. Gebiete 58, 41-53 (1981)

[21] P.A. Ferrari, L.R.G. Fontes, Current fluctuations for the asymmetric simple exclusion process. Ann. Probab. 22, 820-832 (1994)

[22] T. Hwa, E. Frey, Exact scaling function of interface growth dynamics. Phys. Rev. A 44, 7873-7876 (1991)

[23] T. Hwa, T. Nattermann, Disorder induced depinning transition. Phys. Rev. B 51, 455-469 (1995)

[24] L.-H. Tang, I. F. Lyuksyutov, Directed polymer localization in a disordered medium. Phys. Rev. Lett. 71, 2745-2748 (1993) 